\title{
The variability of morphological characters and mycelium growth rate of monoconidial culture of Botrytis cinerea Pers.
}

\begin{abstract}
HANNA BRYK
Institute of Pomology and Floriculture, ul. Pomologiczna 18, 96-100 Skierniewice, Poland

(Received: September 26, 1984)

Abstract

Variability in size of conidia, formation of sclerotia and mycelial growth were examined in monoconidial cultures of 5 isolates of Botrytis cinerea Pers. The influence of two media on the above-mentioned features was also studied. It was found that Botrytis cinerea Pers. is relatively homogenous in respect to the size of conidia. Out of the 50 monoconidial cultures tested, only two had conidia somewhat larger than the remaining cultures. Monoconidial cultures derived from the same isolate varied among themselves in the rate of mycelial growth and in the number and size of sclerotia. The kind of medium used influenced mycelial growth and sclerotia formation. Better mycelial growth was observed on PDA medium than on Czapek's medium. On the latter, the fungus produced more sclerotia which were, however, smaller in size than on PDA medium.
\end{abstract}

\section{INTRODUCTION}

The species Botrytis cinerea Pers. (perfect stage Botryotinia fuckeliana (de Bary/Whetzel) is very unhomogeneous genetically ( $\mathrm{H}$ a $\mathrm{n} \mathrm{s}$ e $\mathrm{n}$ and $\mathrm{S} \mathrm{m} \mathrm{it} \mathrm{h,}$ 1932). Many forms, or strains, of this fungus which differ in their morphological characters, growth on artificial media, ability to produce conidia and sclerotia, have been shown to exist ( $\mathrm{P}$ a u l, 1929; G u p t a, 1960; G r i n d l e, 1979). Some authors are of the opinion that the morphological features of this fungus change depending on its environmental and nutritional conditions. $\mathrm{V}$ a $\mathrm{n}$ e $\mathrm{v}$ (1972) showed that the size of conidia changed depending on whether the fungus grew on artificial medium or its natural host.

In the opinion of other authors, Botrytis cinerea Pers. is relatively homogenous in respect to its morphological features ( $\mathrm{G}$ o r l e $\mathrm{n} \mathrm{ko}$ and M a n u rovskay a, 1971; Dubos and B u 1 i t, 1973). 
In most studies, differences in morphological characters among isolates were examined. Data is lacking on the question if morphological characters within an isolate are identical or not.

The aim of this study was to define the degree of variability of morphological characters and mycelium growth-rates of monoconidial culture within five selected isolates of Botrytis cinerea Pers. Also studied was the effect of the type of medium on the size of conidia, mycelial growth and sclerotium formation by this fungus.

\section{MATERIAL AND METHODS}

The study was conducted on five isolates of Botrytis cinerea Pers. differing in their origin and pathogenicity to apples. The method used to determine their pathogenicity is described in a previous paper (B r y k, 1985). The following isolates were used:

no. 11 - isolated from peaches - weakly pathogenic to apples,

no. 20 - isolated from raspberries - moderately pathogenic to apples,

no. 28 - isolated from raspberries - moderately pathogenic to apples,

no. 17 - isolated from raspberries - moderately pathogenic to apples,

no. 9 - isolated from tomatoes - highly pathogenic to apples.

Ten monoconidial cultures were obtained from each isolate using the method of "gradual dilution" of inoculum and additional microscopic observations. Each culture was inoculated on two media - potato-agar (PDA, Difco) and Czapek's mineral medium set with agar. The experiment was run in 5 replications, where a replication was one, $10 \mathrm{~cm}$ in diameter, Petri dish of each of the media. After 3 days of incubation of the cultures in the dark at $20^{\circ} \mathrm{C}$, the growth of the colony was measured by determining their 2 perpendicular diameters. After 14 days, sclerotium formation was evaluated by counting the number of sclerotia on one dish and measuring their lengths and widths. In the microscopic studies, the lengths and widths of the conidia were measured and the length to width ratio (l/w) was calculated, because according to $\mathrm{D} \mathrm{u} \mathrm{b} \mathrm{o} \mathrm{s} \mathrm{and} \mathrm{B} \mathrm{u} 1$ i t (1973), this index is more suitable in studying morphological variability of fungi than the simple dimensions of the spores. One hundred conidia were measured from each monoconidial culture on each medium. The results dealing with growth on two media were analysed statistically using the variance method; averages within each isolate were compared by the Duncan Test.

\section{RESULTS}

The dimensions of conidia from 50 monoconidial cultures of Botrytis cinerea Pers. are given in Table 1. The extremes of length and width are given as well as the range of the $1 / w$ index for each culture. It was found that the variability in 
T a b le 1

The dimensions of conidia of monoconidial cultures of Botrytis cinerea Pers. $(\mu \mathrm{m})$

\begin{tabular}{|c|c|c|c|c|}
\hline \multirow[b]{2}{*}{$\begin{array}{l}\text { Culture } \\
\text { no. }\end{array}$} & \multicolumn{2}{|c|}{ Potato medium (PDA) } & \multicolumn{2}{|c|}{ Czapek's medium } \\
\hline & length $\times$ width & $\begin{array}{c}\text { ratio of } \\
\text { length to width } \\
(1 / w)\end{array}$ & length $\times$ width & $\begin{array}{c}\text { ratio of } \\
\text { length to width } \\
(1 / w)\end{array}$ \\
\hline 1 & 2 & 3 & 4 & 5 \\
\hline 901 & $8.9-11.6 \times 7.4-9.9$ & $1.1-1.5$ & $8.4-11.6 \times 6.8-8.4$ & $1.1-1.4$ \\
\hline 902 & $8.4-11.0 \times 7.4-8.9$ & $1.1-1.4$ & $8.9-11.0 \times 6.8-9.5$ & $1.1-1.5$ \\
\hline 903 & $9.5-12.1 \times 7.4-8.9$ & $1.2-1.6$ & $8.9-11.6 \times 7.4-9.5$ & $1.1-1.5$ \\
\hline 904 & $8.9-11.0 \times 6.8-8.9$ & $1.1-1.4$ & $8.9-11.0 \times 7.4-8.9$ & $1.1-1.5$ \\
\hline 905 & $8.4-11.0 \times 6.8-9.5$ & $1.1-1.5$ & $8.4-11.0 \times 6.8-8.4$ & $1.1-1.5$ \\
\hline 906 & $8.9-12.1 \times 7.4-8.9$ & $1.1-1.5$ & $9.5-11.6 \times 7.4-9.9$ & $1.1-1.4$ \\
\hline 907 & $9.5-12.1 \times 7.4-9.5$ & $1.1-1.5$ & $8.4-11.0 \times 7.4-9.5$ & $1.1-1.4$ \\
\hline 908 & $8.9-11.0 \times 7.4-9.5$ & $1.1-1.4$ & $8.9-11.0 \times 6.8-8.4$ & $1.1-1.4$ \\
\hline 909 & $9.5-12.1 \times 7.4-9.5$ & $1.1-1.5$ & $8.9-11.0 \times 7.4-8.9$ & $1.1-1.4$ \\
\hline 910 & $8.4-11.0 \times 7.4-8.9$ & $1.1-1.4$ & $8.4-11.6 \times 6.8-9.5$ & $1.1-1.4$ \\
\hline 1101 & $8.4-11.1 \times 6.8-9.5$ & $1.0-1.4$ & $8.4-11.1 \times 6.3-8.4$ & $1.0-1.5$ \\
\hline 1102 & $8.4-11.6 \times 6.8-9.5$ & $1.1-1.4$ & $7.9-11.1 \times 6.8-9.5$ & $1.1-1.4$ \\
\hline 1103 & $7.9-11.6 \times 6.8-8.9$ & $1.1-1.4$ & $8.4-11.6 \times 6.8-9.9$ & $1.1-1.4$ \\
\hline 1104 & $7.9-11.1 \times 7.4 .9 .5$ & $1.1-1.4$ & $7.9-10.5 \times 6.8-8.9$ & $1.1-1.4$ \\
\hline 1105 & $8.4-11.1 \times 6.8-9.5$ & $1.1-1.4$ & $8.4-11.1 \times 6.8-9.5$ & $1.1-1.4$ \\
\hline 1106 & $7.9-11.6 \times 6.3-9.5$ & $1.1-1.4$ & $7.9-11.1 \times 6.3-8.9$ & $1.1-1.5$ \\
\hline 1107 & $7.9-10.5 \times 6.8-9.5$ & $1.1-1.4$ & $7.9-11.1 \times 6.3-9.5$ & $1.1-1.5$ \\
\hline 1108 & $8.4-10.5 \times 6.8-9.5$ & $1.1-1.3$ & $8.4-10.5 \times 6.8-8.9$ & $1.1-1.5$ \\
\hline 1109 & $8.4-11.6 \times 6.8-9.5$ & $1.1-1.4$ & $8.4-10.5 \times 6.8-9.9$ & $1.0-1.4$ \\
\hline 1110 & $8.4-10.5 \times 6.8-9.5$ & $1.1-1.4$ & $8.4-10.5 \times 6.8-8.9$ & $1.1-1.5$ \\
\hline 1701 & $8.4-11.4 \times 7.4-8.9$ & $1.1-1.4$ & $8.4-11.0 \times 6.8-9.5$ & $1.1-1.4$ \\
\hline 1702 & $8.4-10.5 \times 7.4-8.9$ & $1.1-1.4$ & $8.4-10.5 \times 6.8-9.5$ & $1.1-1.4$ \\
\hline 1703 & $7.9-11.0 \times 6.8-8.9$ & $1.1-1.5$ & $7.9-10.5 \times 6.8-8.9$ & $1.0-1.4$ \\
\hline 1704 & $8.4-10.5 \times 6.8-8.4$ & $1.1-1.4$ & $8.4-10.5 \times 7.8-9.5$ & $1.0-1.3$ \\
\hline 1705 & $7.4-10.5 \times 7.4-8.4$ & $1.0-1.4$ & $8.4-11.6 \times 7.4-9.9$ & $1.0-1.5$ \\
\hline 1706 & $8.4-10.5 \times 7.4-9.5$ & $1.1-1.3$ & $8.9-11.6 \times 7.9-10.5$ & $1.0-1.4$ \\
\hline 1707 & $8.4-11.0 \times 7.4-8.9$ & $1.1-1.4$ & $8.4-11.0 \times 7.9-9.5$ & $1.0-1.3$ \\
\hline 1708 & $8.9-11.0 \times 7.4-9.5$ & $1.1-1.4$ & $8.4-10.5 \times 7.4-8.9$ & $1.0-1.4$ \\
\hline 1709 & $8.4-11.6 \times 7.4-9.5$ & $1.1-1.4$ & $8.9-11.6 \times 7.4-9.5$ & $1.2-1.5$ \\
\hline 1710 & $8.4-11.6 \times 7.4-9.5$ & $1.1-1.5$ & $8.4-11.6 \times 6.8-8.4$ & $1.1-1.5$ \\
\hline 2001 & $8.4-10.5 \times 7.4-8.9$ & $1.1-1.4$ & $8.4-10.5 \times 7.4-9.5$ & $1.1-1.3$ \\
\hline 2002 & $7.9-10.5 \times 7.4-8.9$ & $1.0-1.3$ & $8.4-11.1 \times 7.9-10.5$ & $1.0-1.3$ \\
\hline 2003 & $8.4-11.6 \times 7.4-9.5$ & $1.0-1.3$ & $8.9-11.1 \times 7.4-8.9$ & $1.1-1.4$ \\
\hline 2004 & $8.4-11.1 \times 7.4-8.9$ & $1.1-1.4$ & $8.4-11.1 \times 7.4-9.5$ & $1.0-1.5$ \\
\hline 2005 & $8.9-11.6 \times 7.4-9.5$ & $1.1-1.4$ & $8.9-11.6 \times 7.4-9.5$ & $1.1-1.3$ \\
\hline 2006 & $8.4-10.5 \times 7.4-8.9$ & $1.0-1.3$ & $8.4-11.6 \times 7.4-9.5$ & $1.1-1.5$ \\
\hline 2007 & $8.4-11.1 \times 7.4-9.5$ & $1.0-1.4$ & $8.9-12.1 \times 7.4-9.5$ & $1.0-1.5$ \\
\hline
\end{tabular}


Table 1 cont.

\begin{tabular}{cllll}
\hline 1 & \multicolumn{1}{c}{2} & 3 & \multicolumn{1}{c}{4} & 5 \\
\hline 2008 & $8.4-10.5 \times 6.8-8.9$ & $1.0-1.5$ & $8.4-11.1 \times 8.4-9.5$ & $1.0-1.3$ \\
2009 & $8.4-11.1 \times 7.4-8.9$ & $1.1-1.3$ & $8.4-11.1 \times 7.4-9.5$ & $1.0-1.4$ \\
2010 & $9.9-14.2 \times 7.9-11.6$ & $1.1-1.4$ & $7.9-11.6 \times 7.4-10.5$ & $1.0-1.4$ \\
2801 & $8.9-13.7 \times 7.9-10.5$ & $1.0-1.3$ & $8.9-11.6 \times 7.9-10.5$ & $1.1-1.4$ \\
2802 & $8.9-11.1 \times 6.8-8.9$ & $1.1-1.4$ & $9.5-11.6 \times 7.9-10.5$ & $1.0-1.3$ \\
2803 & $8.4-11.6 \times 7.4-9.5$ & $1.0-1.4$ & $8.4-11.6 \times 7.4-9.5$ & $1.1-1.4$ \\
2804 & $8.4-10.5 \times 6.8-8.4$ & $1.1-1.3$ & $8.4-11.6 \times 7.4-9.5$ & $1.1-1.3$ \\
2805 & $7.9-11.1 \times 7.4-9.5$ & $1.0-1.4$ & $8.4-10.5 \times 7.4-9.5$ & $1.1-1.4$ \\
2806 & $8.9-11.6 \times 7.4-8.9$ & $1.1-1.4$ & $8.4-11.6 \times 7.4-9.5$ & $1.1-1.4$ \\
2807 & $8.4-11.6 \times 7.4-9.5$ & $1.1-1.4$ & $8.4-11.1 \times 7.4-8.9$ & $1.1-1.4$ \\
2808 & $8.9-11.6 \times 7.4-9.5$ & $1.1-1.3$ & $8.4-11.1 \times 7.4-8.9$ & $1.1-1.3$ \\
2809 & $8.9-11.1 \times 7.4-8.9$ & $1.1-1.4$ & $8.4-11.6 \times 7.4-8.9$ & $1.1-1.4$ \\
2810 & $8.9-12.1 \times 7.9-9.5$ & $1.1-1.5$ & $8.9-11.1 \times 7.4-9.5$ & $1.0-1.4$ \\
\hline
\end{tabular}

conidial size, both within the studied isolates and among them, is small. The size range of spores is similar. Only cultures no. 2010 and 2801 on PDA medium formed slightly larger conidia. The dimensions of the spores from culture no. 2010 oscillated in the range from 9.9-14.2 $\times 7.9-11.6 \mu \mathrm{m}$, and culture no. 2801 $-8.9-13.7 \times 7.9-10.5 \mu \mathrm{m}$. The type of medium had no effect on the size of the conidia of the remaining cultures.

The $1 /$ w index oscillated between 1.0 and 1.5; only in one case - culture no. 903 - was it 1.2-1.6. The index range of 1.0 to 1.5 shows that both round and elliptic spores were found.

Table 2 presents the colony diameters of the studied fungus cultures after 3 days of incubation. Monoconidial cultures within one isolate differed in their rate of growth on the medium. The least variability in this respect was shown by cultures from isolate no. 20, statistical analysis delineated 2 classes of significance on PDA medium and 3 on Czapek's medium. A decided effect on the rate of mycelium growth by medium type was found. As a rule, cultures grew more rapidly on PDA, only one culture - no. 904 - grew equally well on both media. Variability of culture growth-rate within one isolate was greater on Czapek's medium.

The greatest variability of all of the studied characters was found in the number of sclerotia formed. Table 3 gives the average number of sclerotia on the surface of a Petri dish and the length and width of the most numerous sclerotia in a given monoconidial culture. Culture no. 2802 on PDA medium formed several large sclerotia arranged wreath-like in the center of the dish. The sclerotia of the remaining cultures were round or oval in shape. Monoconidial cultures within one isolate differed from each other in the number of sclerotia formed. The greatest variance was seen within isolate no. 17 on Czapek's medium; the fungus 
Ta b le 2

Mycelium growth of monoconidial cultures of Botrytis cinerea Pers. depending on the medium type

\begin{tabular}{|c|c|c|}
\hline \multirow{2}{*}{$\begin{array}{c}\text { Culture } \\
\text { no. }\end{array}$} & \multicolumn{2}{|c|}{$\begin{array}{c}\text { Diameter of the mycelium (in } \mathrm{mm} \\
\text { on medium }\end{array}$} \\
\hline & Czapek's & PDA \\
\hline 1 & 2 & 3 \\
\hline 907 & $26.2 \mathrm{a}^{*}$ & $88.5 \mathrm{k}$ \\
\hline 903 & $28.5 \mathrm{a}$ & $63.3 \mathrm{~g}$ \\
\hline 909 & $29.0 \mathrm{ab}$ & $80.0 \mathrm{j}$ \\
\hline 902 & $33.0 \mathrm{~b}$ & $59.0 \mathrm{f}$ \\
\hline 905 & $33.4 \mathrm{~b}$ & $75.1 \mathrm{i}$ \\
\hline 906 & $36.0 \mathrm{bc}$ & $68.5 \mathrm{~h}$ \\
\hline 910 & $39.0 \mathrm{c}$ & $76.9 \mathrm{ij}$. \\
\hline 908 & $40.4 \mathrm{~cd}$ & $74.9 \mathrm{i}$ \\
\hline 901 & $43.5 \mathrm{~d}$ & $74.0 \mathrm{i}$ \\
\hline 904 & $49.8 \mathrm{e}$ & $51.4 \mathrm{e}$ \\
\hline 1110 & $21.1 \mathrm{a}$ & $51.5 \mathrm{e}$ \\
\hline 1105 & $27.4 \mathrm{~b}$ & $61.2 \mathrm{f}$ \\
\hline 1109 & $28.4 \mathrm{~b}$ & 52.0 ef \\
\hline 1102 & $28.5 \mathrm{~b}$ & $56.6 \mathrm{f}$ \\
\hline 1101 & $29.8 \mathrm{bc}$ & $67.6 \mathrm{gh}$ \\
\hline 1104 & $33.9 . \mathrm{c}$ & $67.4 \mathrm{gh}$ \\
\hline 1107 & $36.6 \mathrm{~cd}$ & $67.8 \mathrm{gh}$ \\
\hline 1106 & $39.5 \mathrm{~d}$ & $71.5 \mathrm{~h}$ \\
\hline 1108 & $40.8 \mathrm{~d}$ & $67.3 \mathrm{~g}$ \\
\hline 1103 & 54.2 ef & $66.1 \mathrm{~g}$ \\
\hline 1703 & $22.5 \mathrm{a}$ & $57.7 \mathrm{e}$ \\
\hline 1708 & $27.4 \mathrm{ab}$ & $60.8 \mathrm{e}$ \\
\hline 1706 & $27.5 \mathrm{ab}$ & $53.0 \mathrm{de}$ \\
\hline 1704 & $27.9 \mathrm{ab}$ & $57.0 \mathrm{e}$ \\
\hline 1701 & $28.2 \mathrm{~b}$ & $58.0 \mathrm{e}$ \\
\hline 1710 & 28.9 bc & $59.4 \mathrm{e}$ \\
\hline 1707 & $30.2 \mathrm{bc}$ & $59.2 \mathrm{e}$ \\
\hline 1709 & $33.8 \mathrm{c}$ & $58.6 \mathrm{e}$ \\
\hline 1705 & $34.5 \mathrm{c}$ & $59.5 \mathrm{e}$ \\
\hline 1702 & $50.4 \mathrm{~d}$ & $70.6 \mathrm{f}$ \\
\hline 2010 & $19.7 \mathrm{a}$ & $56.1 \mathrm{~d}$ \\
\hline 2007 & $23.1 \mathrm{ab}$ & $58.3 \mathrm{~d}$ \\
\hline 2003 & $25.4 \mathrm{~b}$ & $59.3 \mathrm{~d}$ \\
\hline 2001 & $26.0 \mathrm{~b}$ & $58.4 \mathrm{~d}$ \\
\hline 2005 & $27.5 \mathrm{bc}$ & $55.0 \mathrm{~d}$ \\
\hline 2006 & $28.2 \mathrm{bc}$ & $66.0 \mathrm{e}$ \\
\hline 2002 & $28.3 \mathrm{bc}$ & $59.6 \mathrm{~d}$ \\
\hline
\end{tabular}


Table 2 cont.

\begin{tabular}{cll}
\hline 1. & 2 & 3 \\
\hline 2009 & $28.9 \mathrm{bc}$ & $67.1 \mathrm{e}$ \\
2004 & $32.1 \mathrm{c}$ & $57.9 \mathrm{~d}$ \\
2008 & $33.7 \mathrm{c}$ & $69.0 \mathrm{e}$ \\
2801 & $13.5 \mathrm{a}$ & $39.4 \mathrm{~d}$ \\
2803 & $20.6 \mathrm{~b}$ & $60.8 \mathrm{f}$ \\
2804 & $21.0 \mathrm{~b}$ & $59.9 \mathrm{ef}$ \\
2807 & $22.6 \mathrm{bc}$ & $56.0 \mathrm{e}$ \\
2810 & $24.0 \mathrm{bc}$ & $58.8 \mathrm{ef}$ \\
2802 & $24.2 \mathrm{bc}$ & $58.0 \mathrm{ef}$ \\
2809 & $24.6 \mathrm{bc}$ & $67.1 \mathrm{~g}$ \\
2806 & $26.8 \mathrm{c}$ & $58.2 \mathrm{ef}$ \\
2805 & $27.2 \mathrm{c}$ & $66.5 \mathrm{~g}$ \\
2808 & $30.6 \mathrm{c}$ & $69.5 \mathrm{~g}$ \\
\hline
\end{tabular}

*The significance of differences was evaluated separately for each 10 monoconidial cultures within one starting isolate.

T a b l e 3

Sclerotium formation by monoconidial cultures of Botrytis cinerea Pers. depending on the medium type

\begin{tabular}{|c|c|c|c|c|}
\hline \multirow{2}{*}{$\begin{array}{l}\text { Culture } \\
\text { number }\end{array}$} & \multicolumn{2}{|c|}{ Potato medium (PDA) } & \multicolumn{2}{|c|}{ Czapek's medium } \\
\hline & $\begin{array}{c}\text { no. of } \\
\text { sclerotia }\end{array}$ & $\begin{array}{c}\text { size } \\
(\mathrm{mm})\end{array}$ & $\begin{array}{l}\text { no. of } \\
\text { sclerotia }\end{array}$ & $\begin{array}{c}\text { size } \\
(\mathrm{mm})\end{array}$ \\
\hline 1 & 2 & 3 & 4 & 5 \\
\hline 901 & 58 & $3 \times 3$ & 10 & $3 \times 3$ \\
\hline 902 & 2 & $4 \times 4$ & 20 & $2 \times 2$ \\
\hline 903 & 25 & $3 \times 3$ & 0 & - \\
\hline 904 & 8 & $3 \times 3$ & 15 & $3 \times 3$ \\
\hline 905 & 11 & $3 \times 2$ & 21 & $1 \times 1$ \\
\hline 906 & 0 & - & 0 & - \\
\hline 907 & 6 & $2 \times 2$ & 0 & - \\
\hline 908 & 0 & - & 22 & $3 \times 3$ \\
\hline 909 & 3 & $3 \times 3$ & 37 & $1 \times 1$ \\
\hline 910 & 0 & - & 3 & $2 \times 2$ \\
\hline 1101 & 23 & $4 \times 4$ & 54 & $3 \times 2$ \\
\hline 1102 & 20 & $4 \times 3$ & 64 & $3 \times 3$ \\
\hline 1103 & 34 & $4 \times 3$ & 72 & $3 \times 2$ \\
\hline 1104 & 31 & $4 \times 4$ & 85 & $3 \times 3$ \\
\hline 1105 & 5 & $3 \times 2$ & 39 & $2 \times 2$ \\
\hline 1106 & 22 & $4 \times 4$ & 68 & $3 \times 2$ \\
\hline 1107 & 16 & $4 \times 4$ & 93 & $3 \times 3$ \\
\hline 1108 & 23 & $4 \times 3$ & 53 & $3 \times 3$ \\
\hline
\end{tabular}


Table 3 cont.

\begin{tabular}{|c|c|c|c|c|}
\hline 1 & 2 & 3 & 4 & 5 \\
\hline 1109 & 28 & $4 \times 3$ & 104 & $3 \times 2$ \\
\hline 1110 & 4 & $3 \times 3$ & 24 & $3 \times 3$ \\
\hline 1701 & 26 & $3 \times 3$ & 190 & $2 \times 2$ \\
\hline 1702 & 24 & $3 \times 2$ & 341 & $2 \times 2$ \\
\hline 1703 & 13 & $2 \times 2$ & 9 & $1 \times 1$ \\
\hline 1704 & 2 & $2 \times 3$ & 11 & $2 \times 2$ \\
\hline 1705 & 0 & - & 1 & $2 \times 2$ \\
\hline 1706 & 140 & $2 \times 2$ & 165 & $2 \times 2$ \\
\hline 1707 & 129 & $3 \times 2$ & 148 & $3 \times 3$ \\
\hline 1708 & 93 & $3 \times 3$ & 68 & $2 \times 2$ \\
\hline 1709 & 76 & $3 \times 2$ & 131 & $2 \times 2$ \\
\hline 1710 & 1 & $4 \times 4$ & 45 & $3 \times 2$ \\
\hline 2001 & 16 & $4 \times 3$ & 153 & $2 \times 2$ \\
\hline 2002 & 2 & $3 \times 3$ & 9 & $2 \times 2$ \\
\hline 2003 & 19 & $4 \times 2$ & 13 & $2 \times 2$ \\
\hline 2004 & 0 & - & 4 & $2 \times 2$ \\
\hline 2005 & 8 & $4 \times 3$ & 13 & $3 \times 2$ \\
\hline 2006 & 4 & $5 \times 3$ & 32 & $2 \times 2$ \\
\hline 2007 & 12 & $4 \times 3$ & 12 & $4 \times 3$ \\
\hline 2008 & 0 & - & 6 & $1 \times 1$ \\
\hline 2009 & 3 & $3 \times 2$ & 21 & $3 \times 3$ \\
\hline 2010 & 9 & $4 \times 3$ & 6 & $3 \times 2$ \\
\hline 2801 & 5 & $4 \times 3$ & 6 & $4 \times 3$ \\
\hline 2802 & 1 & $10 \times 10$ & 3 & $2 \times 2$ \\
\hline 2803 & 9 & $4 \times 3$ & 12 & $3 \times 3$ \\
\hline 2804 & 12 & $4 \times 3$ & 9 & $4 \times 3$ \\
\hline 2805 & 4 & $3 \times 3$ & 27 & $2 \times 2$ \\
\hline 2806 & 4 & $5 \times 3^{-}$ & 5 & $2 \times 2$ \\
\hline 2807 & 17 & $4 \times 3$ & 23 & $4 \times 3$ \\
\hline 2808 & 4 & $3 \times 3$ & 36 & $2 \times 2$ \\
\hline 2809 & 4 & $4 \times 3$ & 4 & $3 \times 3$ \\
\hline 2810 & 8 & $5 \times 4$ & 9 & $4 \times 3$ \\
\hline
\end{tabular}

formed from 1 to 341 sclerotia depending on the monoconidial culture. The medium had a significant effect on sclerotium formation. Generally, on Czapek's medium, more numerous and smaller sclerotia were formed than on the potato medium.

\section{DISCUSSION}

Information on the existence of "morphological strains" of the fungus ( $P$ a u l, 1929) as well as on the lack of differences among isolates ( $\mathrm{G}$ o $\mathrm{r}$ l e n- 
$\mathrm{k} \mathrm{o}$ and $\mathrm{Mant} \mathrm{u} \mathrm{rovs} \mathrm{k} \mathrm{y} \mathrm{a,} \mathrm{1971)} \mathrm{is} \mathrm{to} \mathrm{be} \mathrm{found} \mathrm{in} \mathrm{literature.} \mathrm{In} \mathrm{this}$ study, it has been attempted to determine if variability exists among monoconidial cultures within one isolate. On the basis of observations of 50 monoconidial cultures from 5 isolates of the fungus, it can be stated that variability in the size of conidia is small. Only two cultures (no. 2010 and no. 2801) stood out from the others in respect to the size of their conidia. On the other hand, the monoconidial cultures exhibited a high degree of variability in their mycelium growth-rates on artificial media and in sclerotium formation.

On the basis of the experiments carried out here, the conclusion can be drawn that monoconidial cultures of Botrytis cinerea Pers. within one isolate are not homogenous. The reason for this probably is the heterokaryotic nature of Botrytis cinerea Pers. (H a n s e $\mathrm{n}$ and $\mathrm{S} \mathrm{m} \mathrm{it} \mathrm{h,} \mathrm{1932).}$

\section{REFERENCES}

B r y k H., 1985. The pathogenicity of different Botrytis cinerea Pers. isolates to apple and their sensitivity to benzimidazole fungicides. Acta Agrobot. 38: 147-158.

D u b o s B., B u l i t J., 1973. Study of the variability of Botrytis cinerea Pers. isolated from the vine. Botrytis Meeting, Warszawa. Pr. Inst. Sad., Ser. E. nr 2: 1-12.

Gorlenk o M. V., M a n t u r o vis k a y a N. V., 1971. O vnutrividovom raznoobrazii Botrytis cinerea Pers. Tr. Vses. Inst. Zashch. Rast. 29: 52-57.

G r i n d l e M., 1979. Phenotypic differences between natural and induced variants of Botrytis cinerea. J. Gen. Microb. 111: 109-120.

G u p t a S. C., 1960. Two strains of Botrytis cinerea Pers. on Dolichos lablab and Tagetes patula from India. J. Biol. Sci. 3: 92-95.

H a n s e $\mathrm{n} \mathrm{H.} \mathrm{N.,} \mathrm{S} \mathrm{m} \mathrm{i} \mathrm{t} \mathrm{h} \mathrm{R.} \mathrm{E.,} \mathrm{1932.} \mathrm{The} \mathrm{mechanism} \mathrm{of} \mathrm{variation} \mathrm{in} \mathrm{imperfect} \mathrm{fungi:} \mathrm{Botrytis}$ cinerea. Phytopath. 22: 953-964.

P a u 1 W. R. C., 1929. A comparative morphological and physiological study of a number of strains of Botrytis cinerea Pers. with special reference to their virulence. Trans. Br. Mycol. Soc. 14: 118-135.

V a n e v S., 1972. Morphological variability of Botrytis cinerea Pers. under different cultural conditions. (In bulg.) Izv. Bot. Ins. Bulg. Akad. Nauk 22: 193-201.

Zmienność cech morfologicznych i szybkości wzrostu kultur jednozarodnikowych Botrytis cinerea Pers.

Streszczen i e

Badano zmienność cech morfologicznych, tworzenie sklerocjów i szybkość wzrostu grzybni kultur jednozarodnikowych w obrębie 5 wybranych izolatów Botrytis cinerea Pers. Badano także wpływ pożywki na wymienione cechy grzyba. Stwierdzono, że pod względem wielk ości zarodników 
konidialnych Botrytis cinerea Pers. jest względnie jednorodny. Tylko dwie kultury (na 50 badanych) charakteryzowały się nieco większymi zarodnikami od pozostałych.

Kultury jednozarodnik owe, wywodzące się z jednego izolatu, różniły się szybk ością wzrostu na pożywkach oraz tworzeniem sklerocjów. Pożywka ziemniaczana (PDA) była lepsza dla wzrostu większości kultur niż pożywka Czapka. Z kolei na pożywce Czapka kultury tworzyły liczniejsze i drobniejsze sklerocja niż na PDA. 